\title{
Du global au particulier ou comment lire efficacement des documents scientifiques
}

Hélène Marchand

\section{(2) OpenEdition}

Journals

Édition électronique

URL : http://journals.openedition.org/asp/4161

DOI : 10.4000/asp.4161

ISSN : 2108-6354

Éditeur

Groupe d'étude et de recherche en anglais de spécialité

Édition imprimée

Date de publication : 1 février 1994

Pagination : 13-20

ISSN : 1246-8185

Référence électronique

Hélène Marchand, « Du global au particulier ou comment lire efficacement des documents scientifiques », ASp [En ligne], 3 | 1994, mis en ligne le 22 janvier 2014, consulté le 03 mai 2019. URL http://journals.openedition.org/asp/4161; DOI : 10.4000/asp.4161

Ce document a été généré automatiquement le 3 mai 2019.

Tous droits réservés 


\title{
Du global au particulier ou comment lire efficacement des documents scientifiques
}

\author{
Hélène Marchand
}

1 Apprendre à lire des textes scientifiques à des étudiants non spécialistes d'anglais est avant tout un objectif d'autonomie : en effet, l'étudiant confronté à des textes - du mode d'emploi à la communication scientifique spécialisée - doit apprendre à adapter sa stratégie de lecture au type de document qu'il doit lire.

2 Si l'on va du document le plus technique, notice technique par exemple, au document le plus général, article d'information scientifique pour grand public, l'étudiant doit, dans le premier cas, reconnaître d'abord l'ensemble des structures syntaxiques puis connaître les sens des verbes d'action et le lexique spécialisé ; il s'agit d'une lecture linéaire. Dans le deuxième cas, il doit reconnaître d'abord les différentes parties du discours et ses articulations, puis la structure syntaxique des phrases qui auront été sélectionnées comme essentielles pour le sens de l'article, enfin en reconnaître le sens; il s'agit d'une lecture globale.

3 C'est à ce deuxième type de document et à une stratégie de lecture adéquate que nous nous attacherons, pour les raisons suivantes:

- la lecture linéaire d'un texte est la démarche à laquelle l'étudiant a été le plus souvent confronté dans son apprentissage, ce n'est donc pas celle que l'on doit privilégier.

- la lecture globale fait appel à des compétences linguistiques nécessaires à la lecture de textes appartenant à n'importe quelle discipline scientifique.

- L'étudiant aura besoin d'une quantité d'information qu'il ne pourrait pas mémoriser s'il la lisait in extenso.

4 Il nous apparaît donc important de développer cet apprentissage qui sera « rentabilisé » au cours de sa vie professionnelle.

5 On peut définir plusieurs étapes qui permettent une lecture efficace, c'est-à-dire acquérir un maximum d'information dans un minimum de temps. 


\section{Trier, sélectionner l'information}

Il s'agit de faire une lecture rapide pour ne garder que les documents qu'on voudra lire. Cette recherche documentaire n'est pertinente que par rapport à une bonne définition de l'information recherchée, à la connaissance d'une typologie des différentes revues scientifiques et par rapport à la reconnaissance des indices qui les caractérisent.

\section{Analyser le contenu, trouver les mots clefs, faire le plan en donnant des titres informatifs}

7 Les idées, le thème, les concepts, tout ce qui relève du contenu sont en particulier portés par le lexique qu'on peut repérer par des indices non linguistiques et linguistiques. Il s'agit ici d'une lecture globale ; il n'est pas question de lire le texte dans son intégralité mais de faire une lecture « en écrémage ». Ce type de lecture n'est efficace qu'en fonction de la capacité de l'étudiant à anticiper sur l'information à lire, ce qu'il fera d'autant mieux que la première étape de tri de l'information aura été franchie: plus il s'attendra à trouver une information, plus vite il la localisera.

8 Exemples d'indices non linguistiques : changement de typographie ; longueur relative des paragraphes; longueur des commentaires accompagnant les illustrations; types des illustrations.

9 Exemples d'indices linguistiques: les sous-titres; la première et dernière phrase; les titres donnés aux illustrations ; les mots répétés ; les mots appartenant au même champ lexical ; les premiers mots des paragraphes.

\section{Analyser la démarche, reconnaître les différents paragraphes, leurs natures et leurs relations les uns par rapport aux autres}

Il ne suffit pas de comprendre les mots-clefs, encore faut-il les organiser ainsi que les différentes parties du texte $:$ il faut alors repérer, s'il y en a, les articulateurs du discours, "thought connectives», tout ce que l'auteur exprime pour nous faire comprendre sa démarche. L'auteur indique les différentes parties de son discours ${ }^{1}$ par des changements de paragraphes qu'on peut appeler visuels (changements typographiques, espaces, alinéas...). Il peut aussi rendre ce découpage redondant en donnant au lecteur des repères linguistiques; en ce cas, les paragraphes linguistiques coïncideront avec les paragraphes visuels ou même les subdiviseront.

11 On peut définir un «thought connective »comme suit: une unité linguistique plus grande que la phrase, ayant une cohérence et une unité de sens par rapport à l'exposition générale du discours, et obligatoirement introduite par un articulateur logique qui décrit la démarche de l'auteur et jalonne sa forme d'exposition.

$\mathrm{Du}$ point de vue syntaxique, ces "thought connectives" peuvent être des mots ou expressions, mais aussi des constructions complexes et indépendantes. On peut définir un articulateur de paragraphe comme suit : il n'introduit ou n'accompagne pas un mot dans 
la phrase ; (ce n'est pas une préposition); il ne régit pas une proposition; (ce n'est pas une conjonction de subordination); il peut être isolé de la phrase sans la rendre agrammaticale, il peut être isolé de la phrase sans en modifier son sens, il modifie le rapport entre des phrases ou groupes de phrases.

Ainsi, « in spite of » et "though » ne sont pas des "thought connectives» alors que " nevertheless" en est un, bien que ces trois items indiquent une restriction, une contradiction : il est utile d'insister sur cette différence car les distinctions sémantique et grammaticale ne coïncident pas.

Les fonctions des thought connectives sont les suivantes:

1. L'auteur donne des repères chronologiques.

2. L'auteur donne des repères pour découper son propre discours.

3. L'auteur apporte une précision, une explication, ajoute un élément d'information, un exemple.

4. L'auteur réduit son point de vue, apporte une restriction, se contredit, oppose des arguments.

5. L'auteur conclut (définitivement ou provisoirement) donne un jugement, déduit, exprime une conséquence, résume son propos, récapitule.

Les «thought connectives" ont pour but d'aider le lecteur à repérer le plus rapidement possible la démarche d'exposition indiquée par l'auteur, avant même d'aborder le contenu du texte, et ainsi être guidé dans la compréhension de ce contenu. Ils ont également pour but de fournir à celui-ci un corpus utilisable pour l'expression écrite quel que soit le domaine scientifique dont il traite.

Exemples de temps chronologiques modèles. Modèle : In the past, now, in the future

- Looking back over the last 50 years or so,

- in a preliminary experiment

- Previous works have suggested that...

- The present data obtained confirm earlier observations that...

Exemples de temps du discours. Modèle : First, then, finally...

- From table 2, it can be seen that...

- It was observed in the course of the demonstration that...

- For purposes of analysis, the phenomena will be examined separately in the course of the chapter.

Exemples de précision, rajout. Modèle : Moreover

- Another argument is that...

- The observations reported raise a question :

- Its general validity can be demonstrated as follows :

19 Exemples de contradiction, restriction. Modèle : Nevertheless

- Contrary to what has been written,

- Although it is easy to demonstrate,

- It goes without saying that...

Exemples de conclusion, résumé, jugement. Modèle : To conclude,

- The most obvious consequence is that...

- The present investigation has clearly demonstrated that...

- From these hypotheses several predictions can be made :

- The fact demonstrated by this experiment can be put into a general statement :

21 Il est à noter que les étapes 2 et 3 sont en fait 2 étapes solidaires. 
Contenu et démarche sont à analyser dans leur interaction ; une même phrase aura une portée différente selon qu'elle sera exemple, définition, conclusion, etc.

\section{Comprendre les passages sélectionnés}

23 Faire un tri permet de gagner du temps puisqu'on n'a pas besoin de lire de façon linéaire ce qu'on élimine. Paradoxalement, lire efficacement, c'est accepter de ne pas tout lire ; ainsi, les exemples, les citations représentent des passages à rejeter: les indices qui permettent de les reconnaître sans les lire sont en particulier, la ponctuation, les thought connectives, les dates, les noms propres, etc.

Une fois les passages clefs sélectionnés, il faut reconnaître la structure syntaxique de la phrase et comprendre le lexique par rapport aux contraintes syntaxiques: même si un mot est inconnu, il faut d'abord déduire la nature de ce mot et sa fonction, puis avoir recours éventuellement au dictionnaire.

En effet les structures syntaxiques de l'anglais sont en nombre fini: une phrase agrammaticale est incompréhensible même si le lexique est connu car la possibilité d'agencement entre les concepts portés par le lexique est multiple. À l'inverse, une phrase dont on pourra reconnaitre la syntaxe avec des mots de vocabulaire inconnus aura un sens lacunaire mais une partie du sens sera possible. Ce sont donc bien des relations syntaxiques et grammaticales qui permettent de deviner le sens des mots inconnus ou au moins d'éliminer un certain nombre de possibilités de sens pour n'en sélectionner qu'un nombre restreint. Dans la compréhension d'une phrase, l'analyse syntaxique et le repérage des contraintes grammaticales précédera la compréhension du vocabulaire et même la favorisera.

Les principaux points d'articulation, charnières de la phrase, sont les prépositions, les conjonctions de subordination et les pronoms relatifs.

L'étudiant doit tous les connaître car il les rencontre dans les textes de n'importe quelle discipline, et ils représentent une classe fermée alors que les mots de vocabulaire sont une classe ouverte.

28 Cette primauté de la structure syntaxique sur le lexique est illustrée par le poème Jabberwocky de L. Caroll (1871). Dans sa traduction française l'étudiant est dans une situation équivalente à celle d'un texte anglais dont il aurait reconnu la structure syntaxique et ignorerait des mots de vocabulaire.

29 Pour conclure, on peut dire que pour être efficace, la lecture de textes scientifiques ne passe pas d'abord par une compréhension linéaire de tout texte présumément utile, mais par la lecture rapide qui opère un tri, puis une lecture globale qui identifie les mots-clefs, les passages clefs et les thought connectives puis enfin, la lecture linéaire et rapprochée des passages essentiels.

On peut ainsi différencier la compréhension du texte de la compréhension du discours qui permet à l'étudiant d'aller à l'essentiel sans même lire l'ensemble du texte qu'il se propose de comprendre.

31 Cette démarche qui consiste à concentrer les espaces de lecture est une fonction essentielle du scientifique dont F. Dagognet (1993) dit que « il faut dégraisser le discours comme on dégraisse le pot au feu ». 


\section{BIBLIOGRAPHIE}

Dagognet, François. 1993. « Journées du Patrimoine écrit scientifique » octobre 1993.

Caroll, Lewis. 1871. Through the Looking Glass.

\section{NOTES}

1. L'expression «mot d'articulations » est impropre car la notion de mot est la plupart du temps inopérante dans ce cas: nous préférons la notion de thought connective qui fait référence à l'agencement des différents arguments plutôt qu'au découpage de la langue en mots.

\section{RÉSUMÉS}

Cette communication présente une problématique de lecture qui privilégie l'approche globale du texte sur une lecture linéaire. Seront exposés les critères objectifs et les exercices pratiques qui permettent à l'étudiant de sélectionner l'essentiel sans avoir à tout lire.

This paper presents an approach to reading and focuses on the global understanding of a text. objective criteria and practical exercises which allow the student to select essential information when reading are established.

INDEX

Mots-clés : lecture, texte scientifique

Keywords : reading, scientific text

\section{AUTEUR}

\section{HÉLÈNE MARCHAND}

Hélène Marchand enseigne à l'Université Claude Bernard Lyon 1. helene.marchand@univlyon1.fr 